\title{
Asteroseismology and rotation in the main sequence
}

\author{
Andressa Jendreieck ${ }^{1}$, Eduardo Janot Pacheco ${ }^{1}$, \\ Laerte B. P. de Andrade ${ }^{1}$ and Juan Carlos Suárez ${ }^{2}$ \\ ${ }^{1}$ Instituto de Astronomia, Geofísica e Ciências Atmosféricas, Universidade de São Paulo, \\ Rua do Matão 1226, 05508-090, São Paulo/SP, Brazil \\ ${ }^{2}$ Instituto de Astrofísica de Andalucía, Glorieta de la \\ Astronomía, E-18008 Granada, Spain
}

\begin{abstract}
In this project, we study the effects of stellar rotation on the pulsation predictions for stars in the Main Sequence following the series $\delta \mathrm{Scu}, \gamma$ Dor, SPB, Be and $\beta$ Cep. The objects' rotation in this series span from a few $\mathrm{km} / \mathrm{s}$ to a few hundreds of $\mathrm{km} / \mathrm{s}$. We will compare theoretical predictions yielded by the codes CESAM/FILOU with published data from the MOST and CoRoT satellites. A better diagnostic of the rotation effects on stellar pulsations will help to improve the oscillatory models.
\end{abstract}

Keywords. stars: oscillations (including pulsations), stars: rotation

\section{Introduction}

The behavior of the oscillations is determined by the cavity where they are formed, giving direct information of the internal structure of the star (Christensen-Dalsgaard, 2003). Non-radial oscillation has been detected in almost all types of stars in many stages of stellar evolution (e.g., Aerts et al., 2008).

The physical nature of the oscillations are either of the nature of standing acoustic waves (commonly referred to as pressure modes or $\mathrm{p}$ modes) or internal gravity waves (g modes). The oscillations modes are described by spherical harmonics with numbers 1 and $\mathrm{m}$ in a radial field with number $\mathrm{n}$ (Unno et al., 1989).

\section{Rotation}

A star with uniforme angular velocity has a coordinate system in the frame rotating with the star $\left(r^{\prime}, \theta^{\prime}, \phi^{\prime}\right)$ related with the coordinates $(r, \theta, \phi)$ in an inertial frame through (e.g., Christensen-Dalsgaard, 2003)

$$
\left(r^{\prime}, \theta^{\prime}, \phi^{\prime}\right)=(r, \theta, \phi-\Omega t)
$$

Thus the frequency is split uniformly according to $\mathrm{m}$ by

$$
\omega_{m}=\omega_{0}+m \Omega
$$

\section{Purpose}

The project aim to study the effect of rotation in the oscillation spectrum predicted theoretically for stars in the main sequence. 


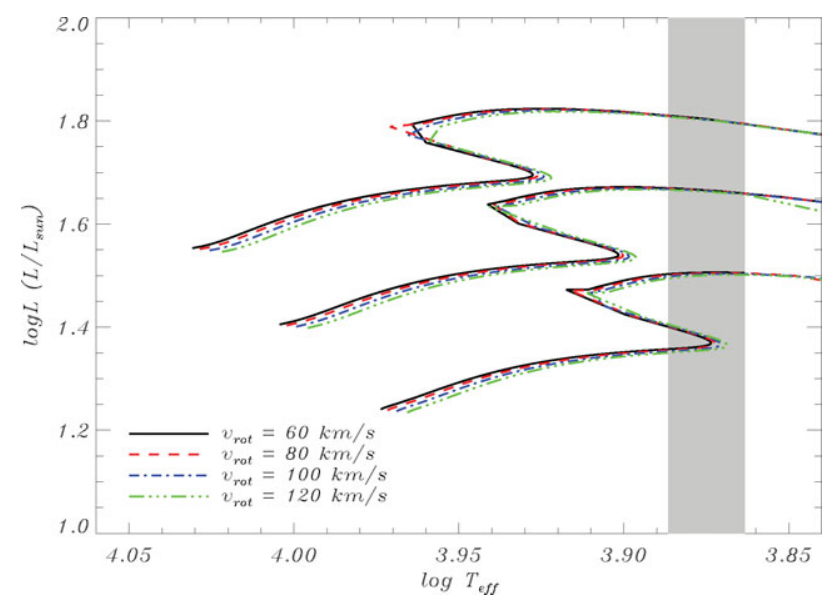

Figure 1. Evolutionary tracks of stars models with masses of $2.0 M_{\odot}$ (bottom lines), $2.2 M_{\odot}$ and $2.4 M_{\odot}$ (upper lines). The grey box represents the uncertainty in effective temperature by Poretti et al. (2009).

\section{Methods}

The theoretical predictions are yielded by the codes

- CESAM (Morel, 1997) that calculate the stellar internal structure and evolution;

- FILOU (Suárez \& Goupil, 2008) that calculate the oscillations frequencies.

\section{Preliminary Results}

The preliminary results are for the $\delta$ Scu star HD 50844 observed with CoRoT and published by Poretti et al. (2009). The evolutionary tracks for a star with masses of 2.0 - $2.4 M_{\odot}$ and rotational velocity $60-120 \mathrm{~km} / \mathrm{s}$ were calculated with CESAM. Figure 1 shows the models that falls inside the uncertainty box. The oscillations frequencies were already calculated with FILOU and are been analyzed.

\section{Future Prospects}

Compare the frequencies calculated for the star HD 50844 with the observations with CoRoT. Do the same analysis with other stars (different masses and rotation velocity) in the main sequence.

\section{Acknowledgements}

I would like to thank IAG-USP, CNPq and FAPESP for financial support in this project.

\section{References}

Aerts, C., Christensen-Dalsgaard, J., Cunha, M., \& Kurtz, D. W. 2008, Solar Phys., 251, 3 Christensen-Dalsgaard, J. 2003, Lectures Notes on Stellar Oscillations (Denmark, Aarhus Universitet), 5th Ed.

Morel, P. 1997, A\&AS, 124, 597

Poretti, E., Michel, E., Garrido, R., \& Lefèvre, L. et al. 2009, A $A$ A, 506, 85

Suárez, J. C. \& Goupil, M. J. 2008, Ap\&SS, 316, 155

Unno, W., Osaki, Y., Ando, H., Saio, H., \& Shibahashi, H. 1989, Nonradial oscillations of stars (Tokyo: University of Tokyo Press), 2nd Ed. 